\title{
Total Ionizing Dose Results and Displacement Damage Results for Candidate Spacecraft Electronics for NASA
}

\author{
Donna J. Cochran ${ }^{1}$, Scott D. Kniffin ${ }^{2}$, Member, IEEE, Kenneth A. LaBel ${ }^{4}$, Member, IEEE, \\ Martha V. O'Bryan ${ }^{3}$, Member, IEEE, Robert A. Reed ${ }^{4}$, Member, IEEE, \\ Ray L. Ladbury ${ }^{2}$, Member, IEEE, James W. Howard Jr. ${ }^{5}, S R$. Member, IEEE, Christian Poivey ${ }^{6}$, \\ Member, IEEE, Stephen P. Buchner ${ }^{1}$, Member, IEEE, Cheryl J. Marshall ${ }^{4}$, Member, IEEE, \\ Paul W. Marshall ${ }^{9}$, Member, IEEE, Hak S. Kim ${ }^{5}$, Donald K. Hawkins ${ }^{4}$, \\ Martin A. Carts ${ }^{3}$, James D. Forney ${ }^{5}$, Anthony B. Sanders ${ }^{4}$, John Bings ${ }^{7}$, John Seiler ${ }^{7}$, \\ Norman E. Hall, ${ }^{8}$, Tim Irwin', Zoran Kahric', Stephen R. Cox ${ }^{4}$, and Christopher Palor ${ }^{2}$
}

1. QSS, Lanham, MD 20706

2. Orbital Sciences Corporation, McLean, VA

3. Raytheon Information Technology \& Scientific Services, Lanham, MD 20706-4392

4. NASA/GSFC, Code 561.4, Greenbelt, MD 20771

5. Jackson \& Tull Chartered Engineers, Washington, D. C. 20018

6. SGT, Greenbelt, MD 20770

7. NAVSEA-Crane Div., Craine, IN 47522

8. JAYCOR, San Diego, CA 92121

9. Consultant

\begin{abstract}
We present data on the vulnerability of a variety of candidate spacecraft electronics to total ionizing dose and displacement damage. Devices tested include optoelectronics, digital, analog, linear bipolar devices, hybrid devices, Analog-toDigital Converters (ADCs), and Digital-to-Analog Converters (DACs), among others.
\end{abstract}

\section{INTRODUCTION}

Commercial and emerging technology devices have $C_{\text {established themselves in the space flight community as }}$ a means to meet the needs for higher performance, cost savings, and scheduling demands. With this dramatic increase in the use of these devices, the importance of ground testing for the effects of total ionizing dose (TID) and protoninduced degradation (also known as displacement damage (DD)) to qualify the devices for flight, has increased due to the often uncertain nature of such devices.

The results of testing presented here were done to establish the sensitivity of the devices selected as candidate spacecraft electronics to TID and proton damage (both ionizing and nonionizing). This testing serves to determine the limit to which a candidate device may be used or if it may not be used at all.

The work presented is sponsored by NASA Electronic Parts and Packaging (NEPP) Program's Electronics Radiation Characterization (ERC) Project, Defense Threat Reduction Agency (DTRA) under IACRO 02-4039I, and NASA Flight Projects.

Donna J. Cochran is with QSS Group Inc., Lanham, MD 20706-4392 (telephone: 301-286-8258, e-mail: donna.cochran@gsfc.nasa.gov).

Scott Kniffin is with Orbital Sciences Corporation, McLean, VA (telephone: 301-286-1185, e-mail: scott.kniffin@gsfc.nasa.gov).

Kenneth A. LaBel is with NASAGSFC, Code 561.4, Greenbelt, MD 20771 USA, (telephone: 301-286-9936, e-mail: kenneth.a.label@.nasa.gov).

Robert A Reed is with NASA/GSFC, Code 561.4, Greenbelt, MD 20771 USA, (telephone: 301-286-2153, e-mail: robert.a.reed@nasa.gov).
For single event effects (SEE) results, see a companion paper submitted to the 2003 IEEE NSREC Radiation Effects Data Workshop entitled: "Single Event Effects Results for Candidate Spacecraft Electronics for NASA" by M. O'Bryan, et al. [1]

\section{TEST TEChNiQues AND SETUP}

\section{A. Test Facilities - TID}

TID testing was performed using a Co-60 source at the Goddard Space Flight Center Radiation Effects Facility (GSFC REF). The source is capable of delivering a dose rate of up to $0.5 \mathrm{rads}(\mathrm{Si}) / \mathrm{s}$, with dosimetry being performed by an ion chamber probe.

Testing performed at Naval Surface Warfare Center Division (NAVSEA) used the Shepherd Model 484 Cobalt- 60 Tunnel Irradiator Test Facility. The source is capable of delivering dose rates between $0.8 \mathrm{rads}(\mathrm{Si}) / \mathrm{s}$ and 49.5 $\operatorname{rads}(\mathrm{Si}) / \mathbf{s}$.

Testing performed by JAYCOR was done at four facilities: The Salk Institute (Salk Inst.) Gammabeam 150-C Co-60 Irradiator, the Sandia National Laboratories (SNL) Gamma Irradiation Facility (GIF), the Defense Microelectronics Activity (DMEA) Science and Engineering Gamma Irradiation Test (SEGIT) Facility using a J.L. Shepherd \& Assoc. Model 81 Co-60 irradiator, and Titan Corp. Pulse Sciences Division (PSD) Cs-137 irradiator. TID facilities are summarized in Table $\mathrm{I}$. 
Table I: Gamma Ray TID Test Facilities Goddard Space Flight Center Radiation Effects Facility (GSFC REF) Co-60 Naval Surface Warfare Center Division (NAVSEA) Shepherd Model 484 Cobalt-60 Tunnel Irradiator Test Facility

JAYCOR: Salk Institute, Sandia National Laboratories, Defense Microelectronics Activity, (Co-60), Titan Corp. (Cs-137)

\section{B. Test Facilities - Proton Damage}

Proton DD/TID tests were performed at two facilities: The University of California at Davis (UCD) Crocker Nuclear Laboratory (CNL) that has a 76" cyclotron (maximum energy of $63 \mathrm{MeV}$ ), and the Indiana University Cyclotron Facility (IUCF) that has an 88" cyclotron (maximum energy of 205 $\mathrm{MeV}$ ). The proton damage test facilities and energies used on the devices are shown in Table II.

Table II: Proton Damage Test Facilities

\begin{tabular}{|l|c|}
\hline \multicolumn{1}{|c|}{ Facility } & $\begin{array}{c}\text { Proton } \\
\text { Energy, } \\
\text { (MeV) }\end{array}$ \\
\hline $\begin{array}{l}\text { University of California at Davis (UCD) Crocker } \\
\text { Nuclear Laboratory (CNL) }\end{array}$ & $26.6-63$ \\
\hline Indiana University Cyclotron Facility (IUCF) & $54-197$ \\
\hline
\end{tabular}

\section{Test Method}

Unless otherwise noted, all tests were performed at room temperature and with nominal power supply voltages.

I) TID Testing

TID testing was performed to the MIL-STD-883 1019.5 test method [2].

2) Proton Damage Testing

Proton damage tests were performed on biased devices with functionality and parametrics being measured either continually during irradiation (in-situ) or after step irradiations (for example, every $10 \mathrm{krad}(\mathrm{Si})$, or every $1 \times 10^{10}$ protons).

\section{TEST RESULTS OVERVIEW}

Abbreviations and conventions are listed in Table III. Abbreviations for principal investigators (PIs) and test engineers are listed in Table IV. Definitions for the categories are listed in Table V. TID results are summarized in Table VI. Displacement Damage testing results are presented in Table VII. Unless otherwise noted, all LETs are in $\mathrm{MeV} \cdot \mathrm{cm}^{2} / \mathrm{mg}$ and all cross sections are in $\mathrm{cm}^{2} /$ device. This paper is a summary of results. Please note that these test results can depend on operational conditions. Complete test reports are available online at http://radhome.gsfc.nasa.gov [3].
Table III: Abbreviations and Conventions:

$A D C$ - analog to digital converter

$\mathrm{CTR}=$ current transfer ratio

DAC - digital to analog converter

$\mathrm{DD}=$ displacement damage

$\mathrm{DNL}=$ differential non-linearity

GPS = global positioning system

GSFC REF = Goddard Space Flight Center Radiation Effects Facility

$\mathrm{H}_{\mathrm{fe}}=$ collector to base current gain

$\mathrm{I}_{\mathrm{b}}=$ input bias current

$I_{d d} I_{c c}=$ power supply current

INL = integral non-linearity

$\operatorname{Krads}(\mathrm{Si})=\operatorname{kilorads}(\mathrm{Si})$

ksps $=$ kilosamples per second

$\mathrm{LDC}=$ lot date code

LET $=$ linear energy transfer $\left(\mathrm{MeV} \cdot \mathrm{cm}^{2} / \mathrm{mg}\right)$

MEMS $=$ Microelectromechanical System

$\mathrm{MeV}=$ Mega Electron Volt

$\mathrm{N} / \mathrm{A}=$ not available

op amp $=$ operational amplifier

$P=$ proton test

$\mathrm{P} / \mathrm{cm}^{2}=$ protons $/ \mathrm{cm}^{2}$

$\mathrm{PI}=$ Principal Investigator

RH $=$ radiation hard

RS $=$ Reed Solomon

SEFI = single event functional interrupt

SEL $=$ single event latchup

TID $=$ total ionizing dose

$V_{i o}=$ input offset voltage

$\mathrm{V}_{\mathrm{ol}}=$ output saturation voltage

$\sigma=$ cross section $\left(\mathrm{cm}^{2} /\right.$ device, unless specified as $\left.\mathrm{cm}^{2} / \mathrm{bit}\right)$

$\sigma_{\mathrm{SAT}}=$ saturation cross section at $\mathrm{LET}_{\max }\left(\mathrm{cm}^{2} /\right.$ device, unless specified as $\mathrm{cm}^{2} / \mathrm{bit}$ )

TABLE IV: LIST OF PRINCIPAL INVESTIGATORS

\begin{tabular}{|c|l|}
\hline Abbreviation & Principal Investigator (PI) \\
\hline SB & Steve Buchner \\
\hline MC & Marty Carts \\
\hline JH & Jim Howard \\
\hline SK & Scott Kniffin \\
\hline RL & Ray Ladbury \\
\hline PM & Paul Marshall \\
\hline CP & Christian Poivey \\
\hline MX & Mike Xapsos \\
\hline
\end{tabular}




\begin{tabular}{|c|c|c|c|c|c|c|c|c|c|c|c|c|c|}
\hline & $\Xi$ & $\overline{5}$ & $\underline{\Xi}$ & $\Xi$ & $\underline{\infty}$ & $\Xi$ & פ & $\Xi$ & $\Xi$ & $\overrightarrow{\stackrel{g}{\Xi}}$ & $\Xi$ & $\begin{array}{l}\stackrel{\sigma}{\varrho} \\
\stackrel{\underline{\omega}}{\Xi}\end{array}$ & $\Xi$ \\
\hline 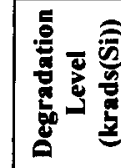 & in & | & : & $\stackrel{\sim}{v}$ & $\bar{v}$ & $\ddot{x}$ & ¿्रे & त्रि & $\frac{\infty}{\vec{V}}$ & $\stackrel{\pi}{\AA}$ & $\underset{\wedge}{\pi}$ & त्र & $\mid \begin{array}{l}\vec{v} \\
\tilde{v} \\
n\end{array}$ \\
\hline & 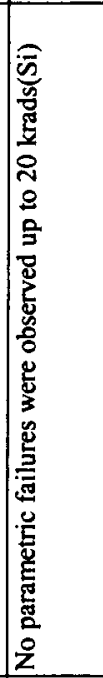 & 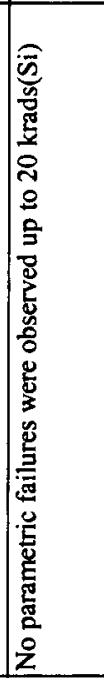 & 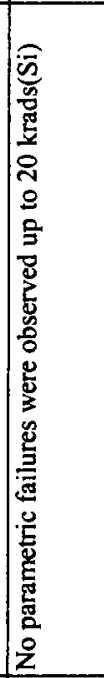 & 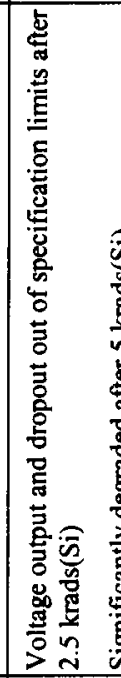 & 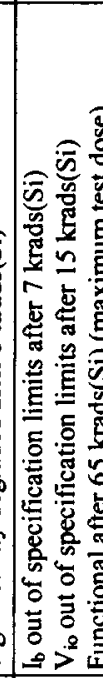 & 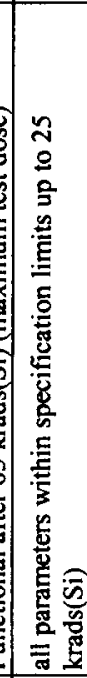 & 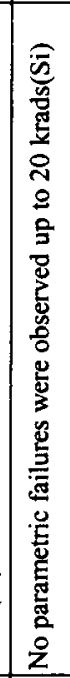 & 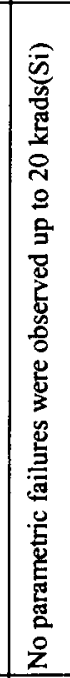 & 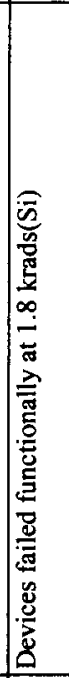 & 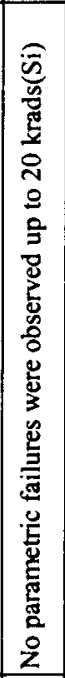 & 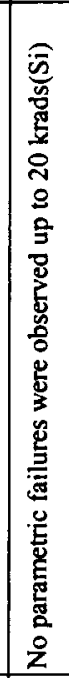 & 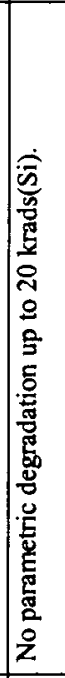 & 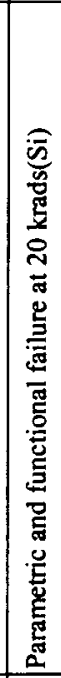 \\
\hline 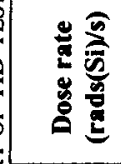 & 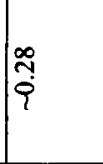 & 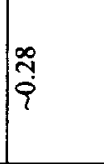 & 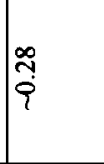 & $\begin{array}{l}f \\
0 \\
0\end{array}$ & \begin{tabular}{|l} 
\\
0 \\
0 \\
0 \\
0 \\
0 \\
0
\end{tabular} & 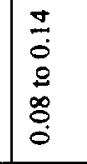 & $\frac{n}{9}$ & $\stackrel{\infty}{\circ}$ & $\overline{\dot{\circ}}$ & $\mid \begin{array}{c}\infty \\
\stackrel{9}{q}\end{array}$ & 文 & $\mid \begin{array}{c}\infty \\
\dddot{q}\end{array}$ & $\overline{\bar{q}}$ \\
\hline 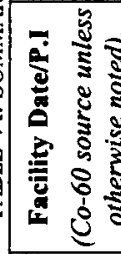 & 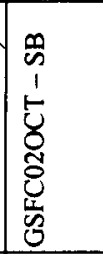 & 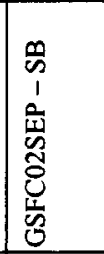 & 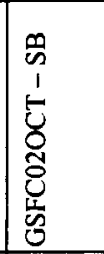 & 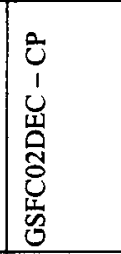 & 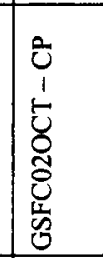 & 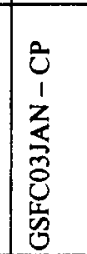 & 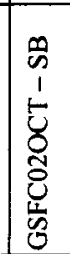 & 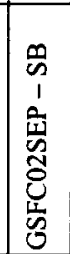 & 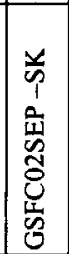 & 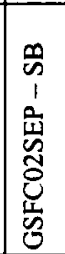 & 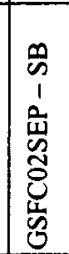 & 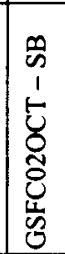 & 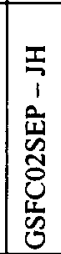 \\
\hline $\bar{\Sigma}$ & 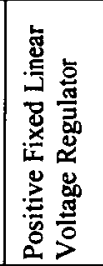 & 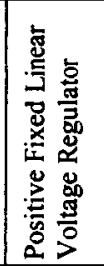 & 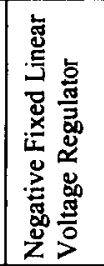 & 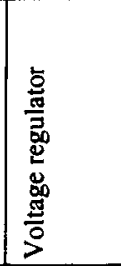 & 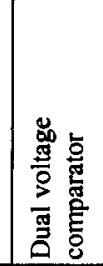 & 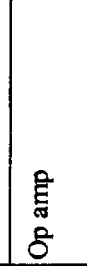 & $\begin{array}{l}\mathbf{E} \\
\mathbf{E} \\
\tilde{8}\end{array}$ & \begin{tabular}{|l}
$\mathbf{E}$ \\
$\mathbf{E}$ \\
$\tilde{g}$
\end{tabular} & 言 & \begin{tabular}{|l}
$\mathbf{g}$ \\
s. \\
s.
\end{tabular} & $\begin{array}{l}\hat{\varepsilon} \\
\tilde{E} \\
\delta \\
\delta\end{array}$ & 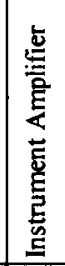 & 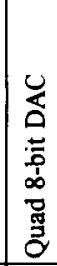 \\
\hline U. & 商 & 商 & 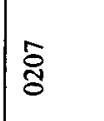 & 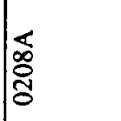 & 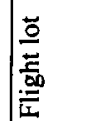 & 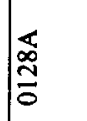 & 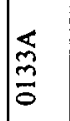 & $\frac{\widehat{N}}{0}$ & $\frac{\infty}{8}$ & $\stackrel{9}{0}$ & $\overline{\bar{\delta}}$ & 芯 & $\overline{\overline{8}}$ \\
\hline$\sum_{\Sigma}^{\frac{\pi}{2}}$ & 言高 & 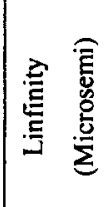 & 言高 & 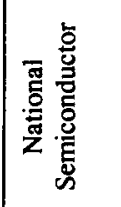 & 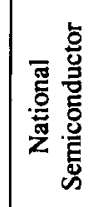 & 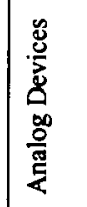 & 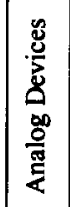 & 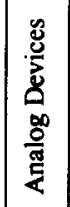 & 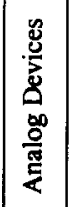 & 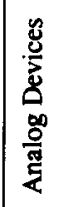 & 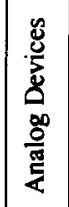 & 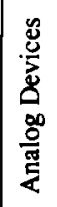 & 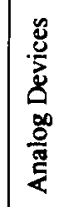 \\
\hline 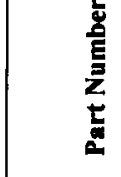 & 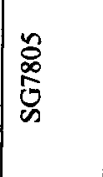 & $\begin{array}{l}\frac{n}{\infty} \\
\vdots \\
\vdots \\
\omega\end{array}$ & $\begin{array}{l}\frac{n}{\alpha} \\
\bar{\delta} \\
\frac{\omega}{n}\end{array}$ & 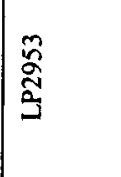 & $\sum_{\Sigma}^{\tilde{\Sigma}}$ & 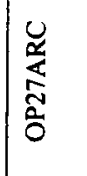 & 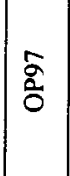 & $\mid \begin{array}{l}\overline{\tilde{\Xi}} \\
\bar{\Xi}\end{array}$ & ठิ & 容 & \begin{tabular}{|l}
$\tilde{\varpi}$ \\
$\frac{\tilde{o}}{\alpha}$
\end{tabular} & 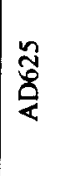 & 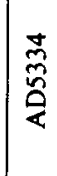 \\
\hline
\end{tabular}




\begin{tabular}{|c|c|c|c|c|c|c|c|c|c|c|c|c|c|}
\hline 这 & $\stackrel{\infty}{\varrho}$ & $\Xi$ & $\mid \begin{array}{l}\overline{\bar{\Xi}} \\
\dot{\overrightarrow{\dot{d}}}\end{array}$ & $\overline{\mathbb{Z}}$ & $\overline{\widetilde{\Omega}}$ & $\frac{\bar{a}}{\frac{\bar{d}}{\bar{d}}}$ & 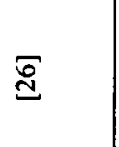 & $\Xi$ & 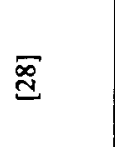 & $\overline{\grave{\Omega}}$ & $\bar{\rho}$ & $\overline{\bar{m}}$ & $\bar{\Xi}$ \\
\hline 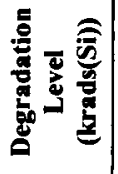 & 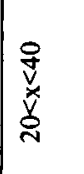 & $\hat{v}$ & $\frac{8}{1}$ & $\vartheta$ & $\tilde{N}$ & 只 & $\tilde{v}$ & $\begin{array}{l}\stackrel{\vec{v}}{v} \\
\ddot{x} \\
\underline{v}\end{array}$ & $\stackrel{\sim}{\vec{v}}$ & $\frac{\rho}{\mathrm{v}}$ & $\vartheta$ & $\vartheta$ & $\mid \begin{array}{l}0 \\
\bar{v} \\
x \\
v \\
v\end{array}$ \\
\hline 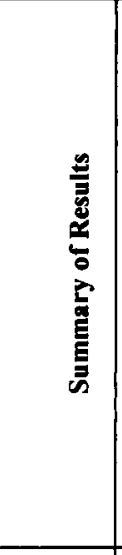 & 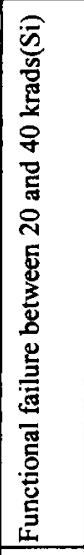 & 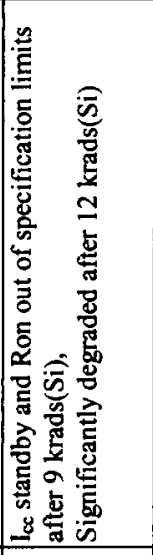 & 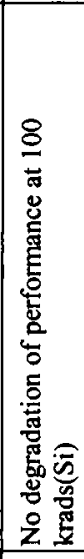 & 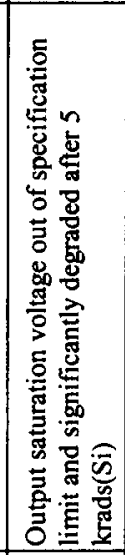 & 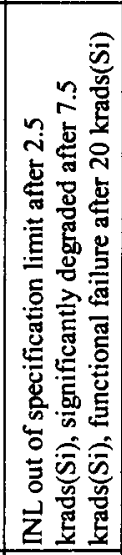 & 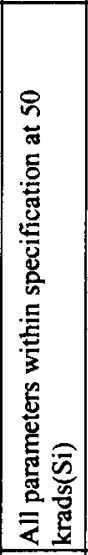 & 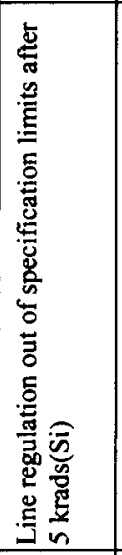 & 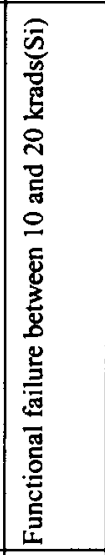 & 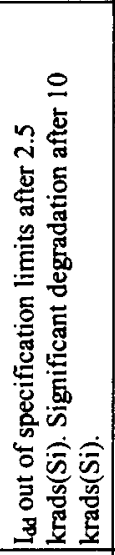 & 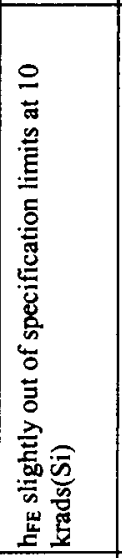 & 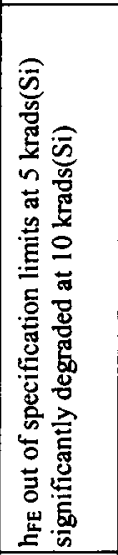 & 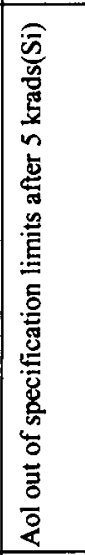 & 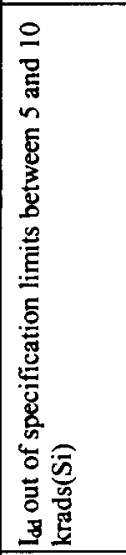 \\
\hline 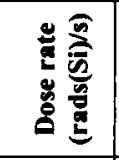 & $\frac{1}{0}$ & \begin{tabular}{|l|} 
\\
0 \\
0 \\
0 \\
0 \\
0 \\
0 \\
0
\end{tabular} & 荌 & ڤั & $\dot{a}$ & $\bar{\varphi}$ & $\bar{q}$ & $\vec{\varphi}$ & $\vec{\varphi}$ & $\begin{array}{l}\bar{a} \\
n \\
\hat{q}\end{array}$ & $\begin{array}{l}\overrightarrow{0} \\
0 \\
0 \\
i\end{array}$ & $\vec{\varphi}$ & $\begin{array}{l}\overrightarrow{8} \\
n \\
0\end{array}$ \\
\hline 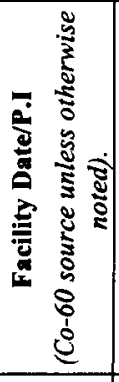 & 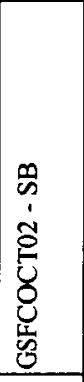 & 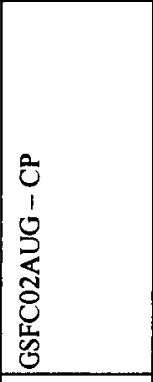 & 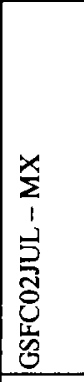 & 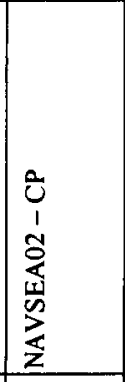 & 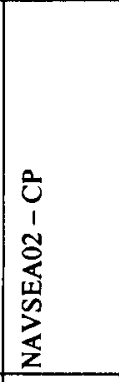 & 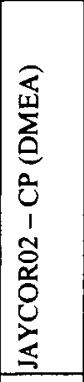 & 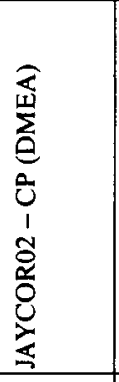 & 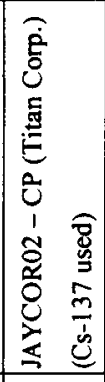 & 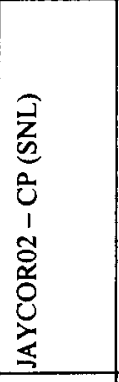 & 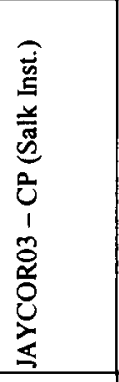 & 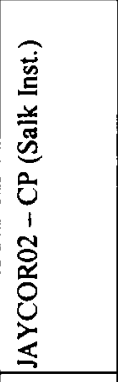 & 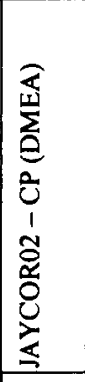 & 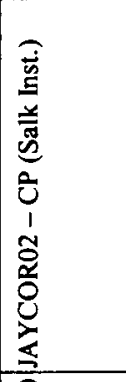 \\
\hline 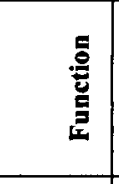 & 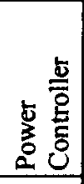 & 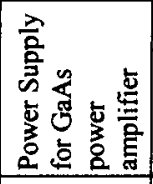 & 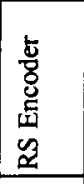 & 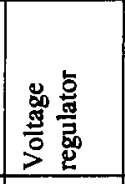 & 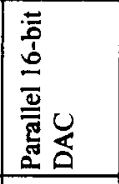 & 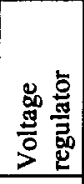 & 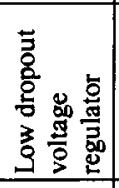 & 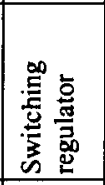 & 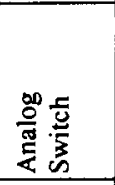 & 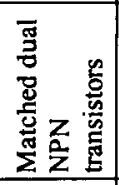 & 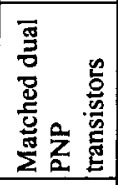 & \begin{tabular}{|l} 
E \\
E్ \\
है \\
\end{tabular} & 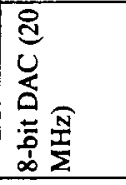 \\
\hline 乌 & $\bar{\Xi}$ & $\overleftarrow{z}$ & 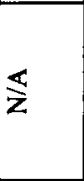 & $\stackrel{\overrightarrow{0}}{0}$ & $\stackrel{\varrho}{0}$ & 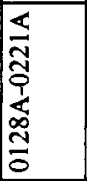 & 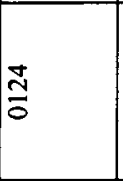 & $\mid \frac{\underline{b}}{\underline{E}}$ & ָे & $\tilde{\tilde{\delta}}$ & & 市 & 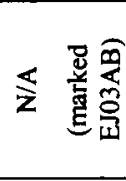 \\
\hline 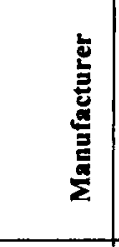 & 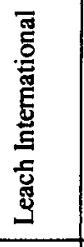 & 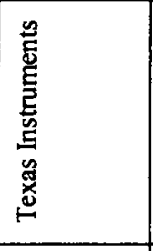 & 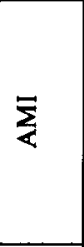 & 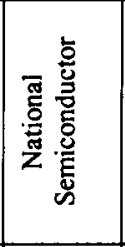 & 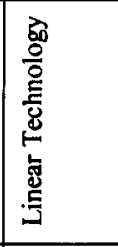 & 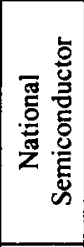 & $\frac{\bar{\nu}}{\Sigma}$ & 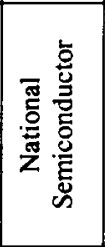 & 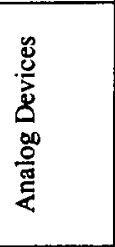 & 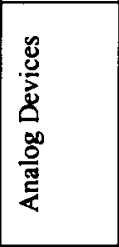 & 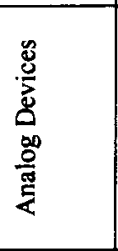 & 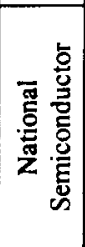 & 兑 \\
\hline 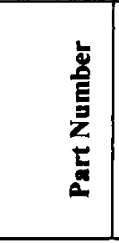 & $\frac{8}{2}$ & $\frac{\hat{\sigma}}{\bar{\alpha}}$ & 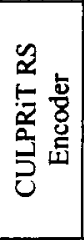 & $\sum_{i=j}^{\rho}$ & $\begin{array}{l}Z \\
0 \\
0 \\
0 \\
0 \\
0 \\
\vdots \\
\end{array}$ & $\begin{array}{l}\bar{\Omega} \\
\stackrel{\Xi}{\Xi} \\
\end{array}$ & 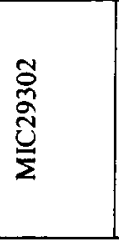 & 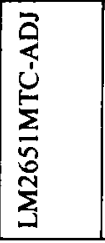 & $\begin{array}{l}\tilde{n} \\
\text { 岁 }\end{array}$ & $\frac{\tilde{Q}}{\stackrel{S}{S}}$ & $\frac{o}{\stackrel{c}{\alpha}}$ & $\frac{7}{0}$ & 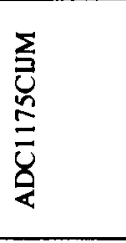 \\
\hline
\end{tabular}




\begin{tabular}{|c|c|c|c|c|c|c|c|c|c|c|c|}
\hline 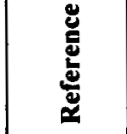 & $\bar{m}$ & 跑 & $\bar{\Xi}$ & $\bar{m}$ & \begin{tabular}{l}
$\overline{0}$ \\
\hdashline
\end{tabular} & $\approx$ & $\stackrel{\infty}{\infty}$ & $\bar{g}$ & 定 & $\bar{\Xi}$ & $\bar{\Xi}$ \\
\hline 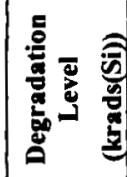 & 8 & $\frac{\varkappa}{\Lambda}$ & 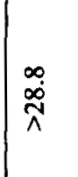 & $v$ & $\eta$ & $\frac{8}{\lambda}$ & $\frac{8}{1}$ & $\stackrel{\vec{z}}{\mathrm{~N}}$ & $\tilde{\varphi}$ & $=$ & g \\
\hline 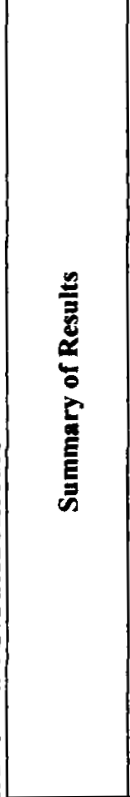 & 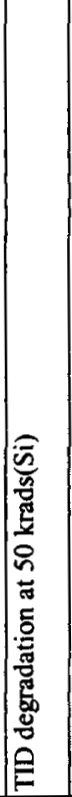 & 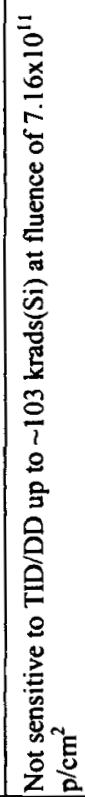 & 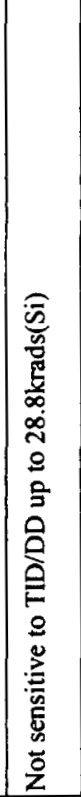 & 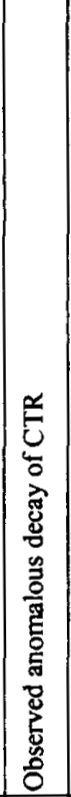 & 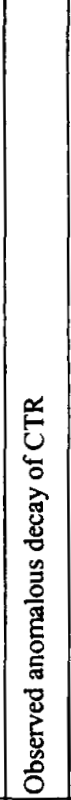 & 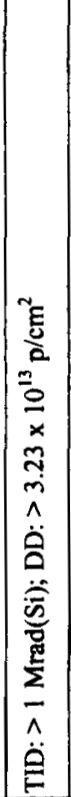 & 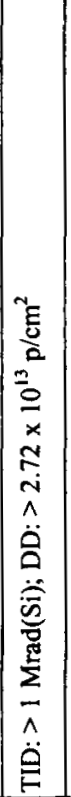 & 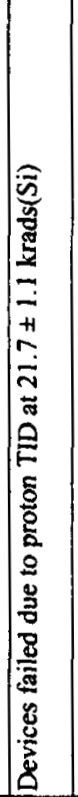 & 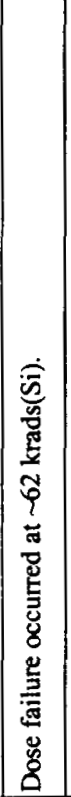 & 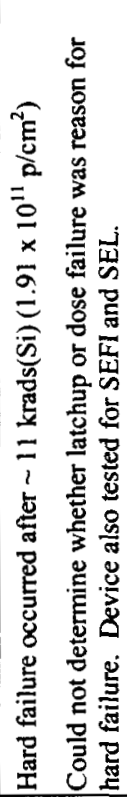 & 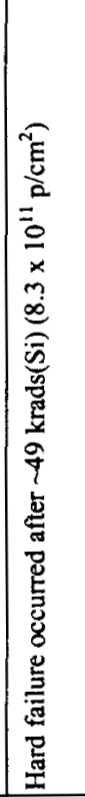 \\
\hline 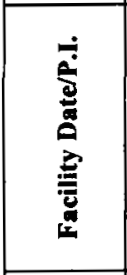 & 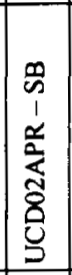 & 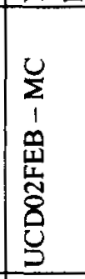 & 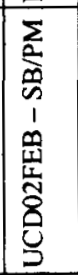 & 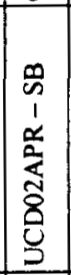 & 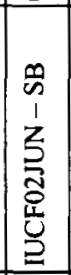 & 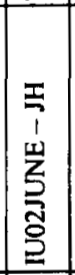 & 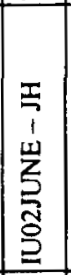 & 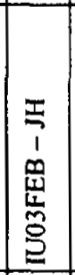 & 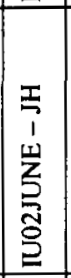 & 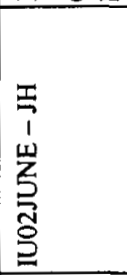 & 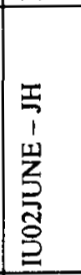 \\
\hline 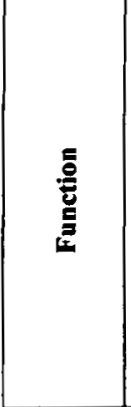 & 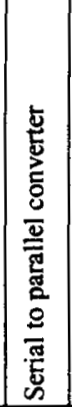 & 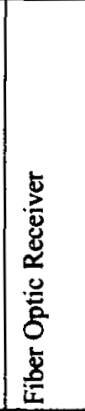 & 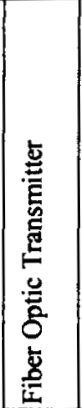 & 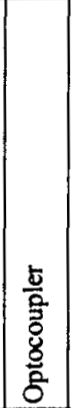 & 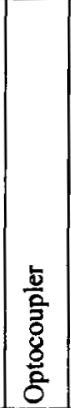 & \begin{tabular}{|l} 
总 \\
要 \\
on
\end{tabular} & 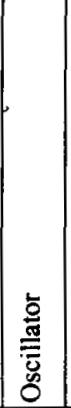 & 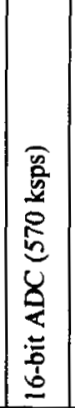 & 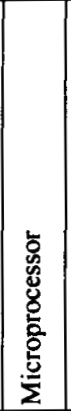 & 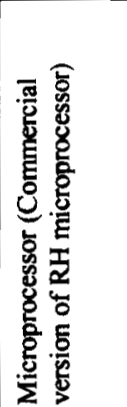 & 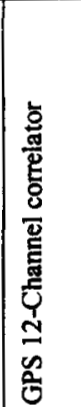 \\
\hline U్ & $\overleftrightarrow{\bar{z}}$ & $\frac{\dot{c}}{m}=$ & $\frac{\dot{m}}{\tilde{z}} \bar{\sigma}$ & ह & है & $\frac{F}{\sigma}$ & $\frac{F}{\partial}$ & $\stackrel{\varrho}{\tilde{\sigma}}$ & בิ & $\leqslant$ & $\$$ \\
\hline 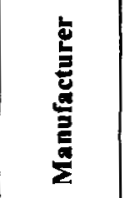 & 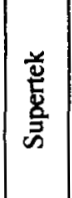 & 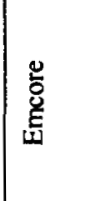 & 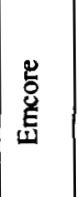 & 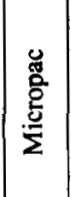 & 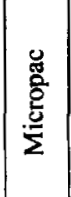 & 量 & $\begin{array}{l}5 \\
\bar{E} \\
\\
\\
\end{array}$ & 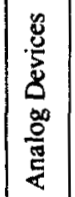 & $\begin{array}{l}\frac{\pi}{\overline{2}} \\
\frac{\bar{g}}{\overline{0}} \\
\frac{1}{\Sigma}\end{array}$ & 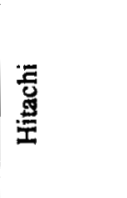 & 总 \\
\hline 点害 & 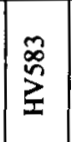 & 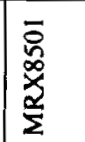 & 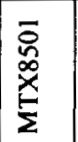 & 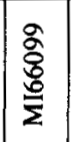 & 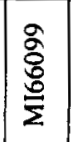 & 号 & $\stackrel{\infty}{\infty}$ & 売 & 该 & $\frac{T}{\omega}$ & $\begin{array}{l}\overline{\widetilde{N}} \\
\text { Sิ }\end{array}$ \\
\hline
\end{tabular}




\section{TID TEST RESULTS AND DISCUSSION}

\section{1) $A D 5334$}

The AD5334 quad 8-bit DAC from Analog Devices was tested to $50 \mathrm{krads}(\mathrm{Si})$ at a dose rate of $\sim 0.11 \mathrm{rads}(\mathrm{Si}) / \mathrm{s}$. Tests were performed at the NASA GSFC REF Co- 60 facility. The devices were biased statically. After $15 \mathrm{krads}(\mathrm{Si})$, all devices began to deviate from the nominal values in DNL, INL, gain error, offset error, and supply current. See Figures 1 and 2 for DNL \& INL results. After $20 \mathrm{krads}(\mathrm{Si})$, the above parameters began to degrade very significantly with DNL and INL having degraded to well outside 8-bit performance.

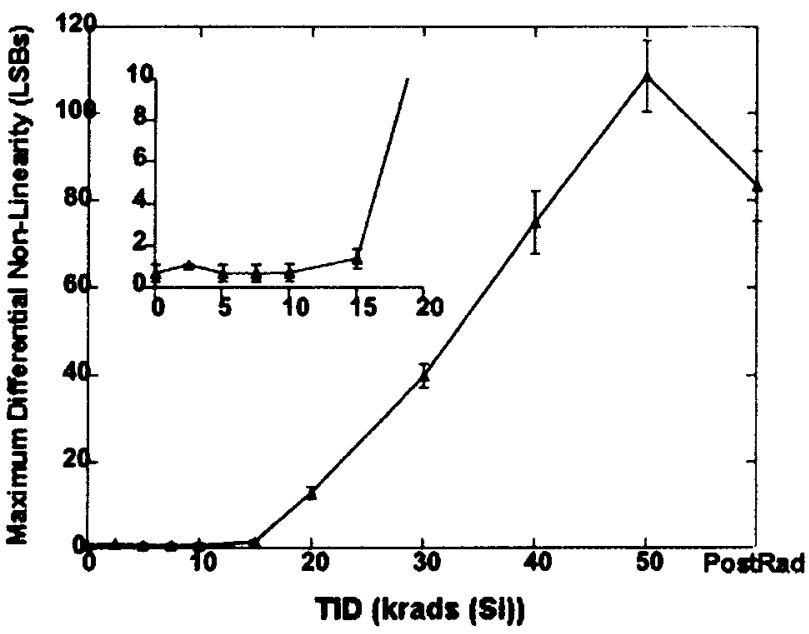

Fig. 1. AD5334 Maximum DNL as a function of TID.

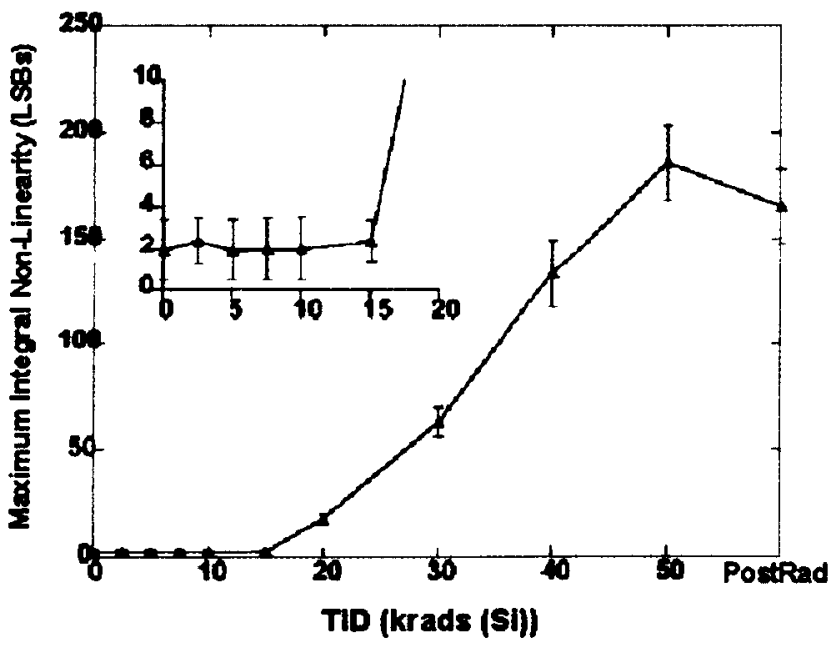

Fig. 2. AD5334 Maximum INL as a function of TID.

After $30 \mathrm{krads}(\mathrm{Si})$, the device lost monotonicity and the output voltage became oscillatory across the entire range of the DAC. Annealing at $100^{\circ} \mathrm{C}$ for one week did not provide significant recovery.

2) $A D G 425$

The ADG425 analog switch from Analog Devices was tested to $50 \mathrm{krads}(\mathrm{Si})$ at an average dose rate of $\sim 0.19$ $\operatorname{rads}(\mathrm{Si}) / \mathrm{s}$. Tests were performed by JAYCOR. Six devices were statically biased and total current was monitored during testing. Two devices were unbiased during testing. After 2.5 $\operatorname{krads}(\mathrm{Si})$, the biased devices were out of specification in $\mathrm{I}_{\mathrm{DD}}$, $I_{S S}, I_{S}$ off $+10 \mathrm{~V}$, and $I_{S \text { off }}-10 \mathrm{~V}$. The degradation between 10 and $20 \mathrm{krads}(\mathrm{Si})$ was significantly enough that the timing and high/low threshold readings could not be made and the devices would not change from state to state. Annealing at room temperature for 1 week resulted in no observed recovery. The unbiased devices show some degradation at 30 and $50 \mathrm{krads}(\mathrm{Si})$. Annealing the unbiased devices resulted in no significant recovery.

3) $L M 193$

The LM193 quad low power voltage comparator from National Semiconductor was tested to $65 \mathrm{krads}(\mathrm{Si})$ at an average dose rate of $\sim 0.25 \mathrm{rads}(\mathrm{Si}) / \mathrm{s}$. Tests were performed at the NASA GSFC REF Co-60 facility. Four devices were statically biased and two devices were unbiased during testing. After $7 \mathrm{krads}(\mathrm{Si})$, both $\mathrm{I}_{b}+$ and $\mathrm{I}_{b}$ - in all devices (biased and unbiased) were well above the specification limits. After $15 \mathrm{krads}(\mathrm{Si}), \mathrm{V}_{\mathrm{IO}}$ were above the specification limit for the four biased devices; one unbiased device went above the specification limit after $35 \mathrm{krads}(\mathrm{Si})$. All other parameters stay within specification limits up to the maximum test dose level of $65 \mathrm{krads}(\mathrm{Si})$ (see Fig. 3.). Annealing at room temperature for 1 week resulted in no significant annealing.

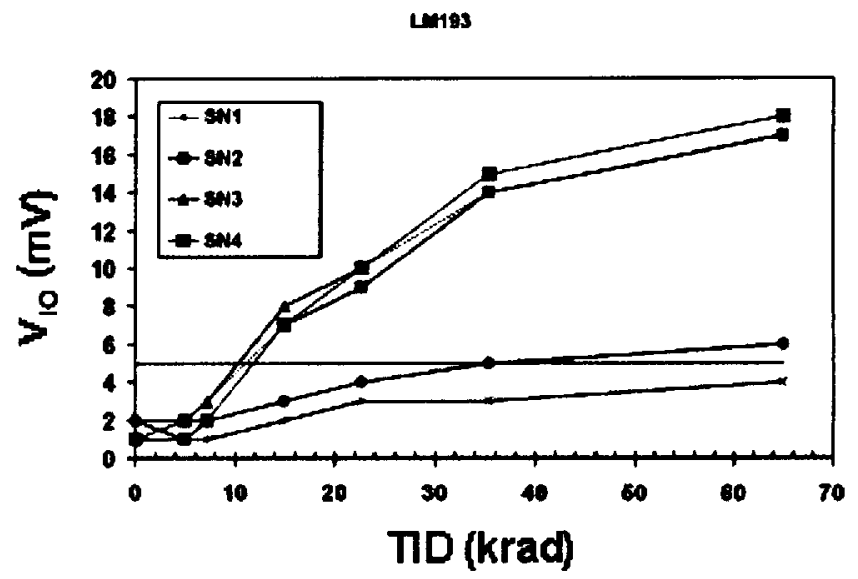

Fig. 3: Variation of $\mathrm{V}_{10}$ with TID in the LM193.

4) $L M 139$

The LM139 (M38510/11201BDA) quad low power voltage comparator from National Semiconductor was tested to $50 \mathrm{krads}(\mathrm{Si})$ at an average dose rate of $0.295 \mathrm{rads}(\mathrm{Si}) / \mathrm{s}$. NAVSEA Crane Division performed the total dose testing at their Co-60 tunnel irradiator. Six devices were statically biased, two unbiased. Results of the total dose testing indicated:

- Both unbiased devices failed to operate properly between $5 \mathrm{krads}(\mathrm{Si})$ and $10 \mathrm{krads}(\mathrm{Si})$ as evidenced by output saturation voltage $\left(\mathrm{V}_{\mathrm{OL}}\right)$ increasing well above specification (See Fig. 4.).

- All statically biased devices failed to operate properly between $10 \mathrm{krads}(\mathrm{Si})$ and $20 \mathrm{krads}(\mathrm{Si})$ as evidenced by output saturation voltage $\left(\mathrm{V}_{\mathrm{OL}}\right)$ increasing well above specification (See Fig. 4). 
- All devices began showing power supply current decrease after $5.0 \mathrm{krads}(\mathrm{Si})$.

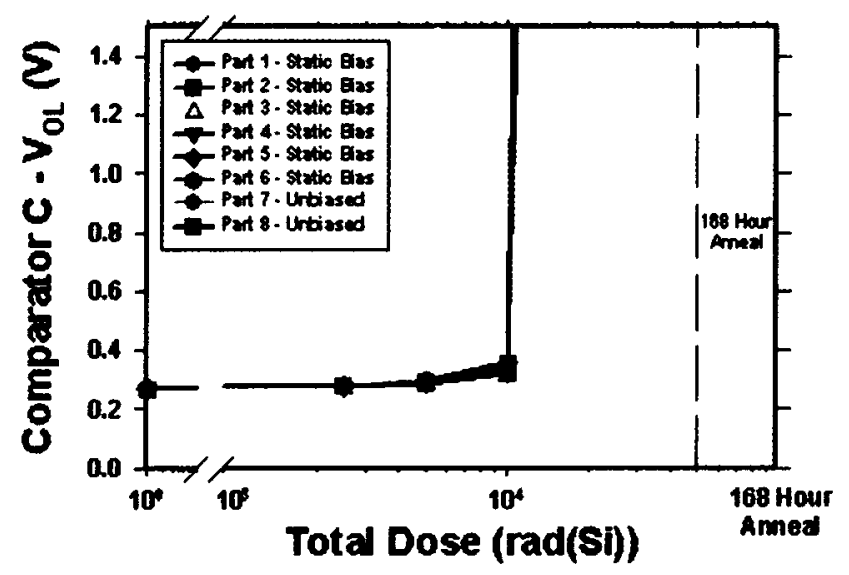

Fig. 4. TID induced $V_{0 L}$ failure of the LMI39.

\section{5) $M R X 850 I / M T X 850 I$}

The MTX8501/MRX8501 $12 \times 1.25$ Gbps parallel array transmitter and receiver modules from Emcore were tested for $\mathrm{DD}$ and TID effects with $63 \mathrm{MeV}$ protons at UC Davis, CNL cyclotron. The first receiver was exposed to a total fluence of $7.16 \times 10^{11}$ protons $/ \mathrm{cm}^{2}$; equivalent to $103 \mathrm{krads}(\mathrm{Si})$. Following this exposure, there was no change in the functioning of the device, or in the current supplied to the board. The second receiver was exposed to a TID of 30.7 $\operatorname{krads}(\mathrm{Si})$ and no change was observed. The transmitter was exposed to a fluence of $2 \times 10^{11}$ protons $/ \mathrm{cm}^{2}$; equivalent to a TID of $28.8 \mathrm{krads}(\mathrm{Si})$ and no change in error rate or operating parameters was observed. These devices were also tested for SEE, those results are also in the test report and are presented in "Single Event Effects Results for Candidate Spacecraft Electronics for NASA" accepted for publication in IEEE NSREC 2003 Data Workshop, July, 2003.

6) $O P 296$

The OP296 micropower, rail-to-rail input and output operational amplifier from Analog Devices was tested to $1.8 \mathrm{krads}(\mathrm{Si})$ at a dose rate of $0.01 \mathrm{rads}(\mathrm{Si}) / \mathrm{s}$. Tests were performed at the NASA GSFC REF Co-60 facility. The devices were biased statically. The devices failed functionally at $1.8 \mathrm{krads}(\mathrm{Si})$ and no recovery was observed after annealing for one week at room temperature.

\section{7) $A D 7664$}

The AD7664 16-bit ADC from Analog Devices was tested with protons at IUCF. While performing other tests, the devices were left in the beam until TID failure. The devices failed at a TID of $21.7+/-1.1 \mathrm{krads}(\mathrm{Si})$. The devices were also tested for proton induced SEL and heavy ion induced SEL see [1] for more details on those tests.

\section{SUMMARY}

We have presented data from recent TID and protoninduced damage tests on a variety of primarily commercial devices. It is the authors' recommendation that this data be used with caution. We also highly recommend that lot testing be performed on any suspect or commercial device.

\section{ACKNOWLEDGMENT}

The Authors would like to acknowledge the sponsors of this effort: NASA Electronics Radiation Characterization (ERC) Project, a portion of NASA Electronic Parts and Packaging Program (NEPP), NASA Flight Projects, and the Defense Threat Reduction Agency (DTRA) under IACRO 02-4039I.

\section{REFERENCES}

[1] M. O'Bryan, et al., "Single Event Effects Results for Candidate Spacecraft Electronics for NASA" submitted to the 2003 IEEE NSREC Radiation Effects Data Workshop.

[2] Department of Defense Test Method Microcircuits, MIL-STD-883 Test Method Standard, Microcircuits, MIL-STD-883 (1000's) (Complete) 6section text, 1000 series test methods, (With all Notices included in Main Document) Dated: 18 December 2000, File name: std883inc1000.pdf, File size: $1414 \mathrm{~kb}$, ppl12-121, http://www.dscc.dla.mil/ Programs/MilSpec/ListDocs.asp?BasicDoc=MIL-STD-883, December 1996.

[3] NASA/GSFC Radiation Effects and Analysis home page, http://radhome.gsfc.nasa.gov

[4] S. Buchner, et al., "Total lonizing Dose Testing: 7805 Positive Voltage Regulator," http://radhome.gsfc.nasa.gov/radhome/ papers/G0902_SG7805.pdf; Oct. 2002.

[5] S. Buchner, et al., "Total lonizing Dose Testing: 7815 Positive Voltage Regulator," http://radhome.gsfc.nasa.gov/radhome/ papers/G0902_SG7815.pdf; Sept. 2002

[6] S. Buchner, et al., "Total Ionizing Dose Testing: 7915 Positive Voltage Regulator," papers/G0902_SG7915.pdf; Sept. 2002

[7] C. Poivey, et al., "LP2953 Test Report," http://radhome.gsfc.nasa.gov/radhome/ papers/G1202_LP2953.pdf; Dec. 2002

[8] C. Poivey, et al., "LM193 TID Test Report," http://radhome.gsfc.nasa.gov/radhome/ papers/ Goct02_LM193.pdf; Oct. 2002

[9] C. Poivey, et al., "OP27 TID Test Report," http://radhome.gsfc.nasa.gov/radhome/ papers/G0103_OP27.pdf; Jan. 2003

[10] S. Buchner, et al., "Total lonizing Dose Testing: of the OP97 LowPower, High-Precision Operational Amplifier," http://radhome.gsfc.nasa.gov/radhome/ papers/G1002_OP97.pdf; Oct. 2002

[11] S. Buchner, et al., "Total Ionizing Dose Testing: OP-221 Dual OpAmp," http://radhome.gsfc.nasa.gov/radhome/ papers/G0902_OP221.pdf; Sept. 2002

[12] S. Kniffin, et al., "Total lonizing Dose (TID)Test Results for the Analog Devices OP296 Op Amp," http://radhome.gsfc.nasa.gov/radhome/papers/G0902_OP296.pdf; Sept. 2002

[13] S. Buchner, et al., "Total lonizing Dose Testing: AD743 Ultralow Noise BIFET Op-Amp," http://radhome.gsfc.nasa.gov/radhome/ papers/G0902_AD743.pdf; Sept. 2002

[14] S. Buchner, et al., "Total lonizing Dose Testing: AD822 Single-Supply, Rail-to-rail, Low Power FET Input Dual Op-Amp," http://radhome.gsfc.nasa.gov/radhome/ papers/G0902_AD822.pdf; Sept. 2002

[15] S. Buchner, et al., "Total lonizing Dose Testing: AD625 Instrument Amplifier," papers/G0902 AD625.pdf; Sept. 2002

[16] S. Buchner, et al., "Total lonizing Dose Testing: AD625 Instrument Amplifier," http://radhome.gsfc.nasa.gov/radhome/ papers/G1002_AD625.pdf; Oct. 2002

[17] J. Howard, et al., "Co-60 Total lonizing Dose (TID) Testing of the Analog Devices AD5334 Digital to Analog Converter (DAC)," 
http://radhome.gsfc.nasa.gov/radhome/ papers/G090302_AD5334.pdf; Sept. 2002

[18] S. Buchner, et al., "Total lonizing Dose Testing (TID) of the P140 Solid State Power Controler (Leach International)," http://radhome.gsfc.nasa.gov/radhome/ papers/G2002_P140.pdf; Oct. 2002

[19] C. Poivey, et al., "TPS9103 TID Test Report," http://radhome.gsfc.nasa.gov/radhome/ papers/G0103_TPS9103.pdf; Jan. 2003

[20] M. Xapsos, et al., "Single Event Effect and Total Ionizing Dose Testingof CULPRiT Reed-Solomon Encoders," http://radhome.gsfc.nasa.gov/radhome/papers/2002_CULPRiT.pdf; Aug. 2003

[21] M. Xapsos, et al., "Evaluation of an Ultra-Low Power Reed-Solomon Encoder for NASA's Space Technology 5 Mission," http://radhome.gsfc.nasa.gov/radhome/papers/G080102_CULPRiT.pdf ; Aug. 2003

[22] J. Seiler, et al, "NAVSEA M38510/11201BDA (LM139) Total Dose Test Report," http://radhome.gsfc.nasa.gov/radhome/ papers/N2002_LM139.pdf; July 2002

[23] J. Bings, et al, "NAVSEA LTC1657 Total Dose Test Report," http://radhome.gsfc.nasa.gov/radhome/ papers/N062002_LTC1657.pdf; June 2002

[24] N. Hall, Sr., et. al, "Total Dose Test Report: LP2951 Micropower Voltage Regulator.," http://radhome.gsfc.nasa.gov/radhome/ papers/DEMA102202_LP2951.pdf; Oct. 2002

[25] N. Hall, Sr., et. al, "Total Dose Test Report: LP2951 Micropower Voltage Regulator.," http://radhome.gsfc.nasa.gov/radhome/ papers/DEMA111902_LP2951_\#2.pdf; Nov. 2002

[26] N. Hall, Sr., et. al, "Total Dose Test Report: MIC29302BT Voltage Regulator.," http://radhome.gsfc.nasa.gov/radhome/ papers/DEMA100802_MIC29302.pdf; Oct. 2002

[27] N. Hall, Sr., et. al, "Total Dose Test Report: LM2651 1.5A Sw. Reg.," http://radhome.gsfe.nasa.gov/radhome/ papers/TIT090302_LM2651.pdf; May 2003

[28] N. Hall, Sr., et. al, "Total Dose Test Report: ADG452BR SPST Switch," http://radhome.gsfc.nasa.gov/radhome/ papers/S080602_ADG452.pdf; Aug. 2002

[29] N. Hall, Sr., et. al, "Total Dose Test Report: MAT-02 Dual NPN Transistor.," papers/S020603_MAT02.pdf; Feb. 2002

[30] N. Hall, Sr., et. al, "Total Dose Test Report: MAT-03 Dual PNP Transistor.," http://radhome.gsfc.nasa.gov/radhome/ papers/S010203 MAT03.pdf; Jan. 2002

[31] N. Hall, Sr., et. al, "Total Dose Test Report: LM6144, 17MHz Rail to Rail Input-Output Op Amp.," http://radhome.gsfc.nasa.gov/radhome/ papers/DM080702_LM6144.pdf; Aug. 2002

[32] N. Hall, Sr., et. al, "Total Dose Test Report: ADC1175 8 Bit A/D Converter," http://radhome.gsfc.nasa.gov/radhome/ papers/S111502_ADC1175.pdf; Nov. 2002

[33] S. Buchner, et al., "Proton Testing of the Supertex HV583 128Channel Serial-to-Parallel Converter With Push-Pull Outputs," http://radhome.gsfc.nasa.gov/radhome/ papers/ D040402_HV583.pdf; Apr. 2002

[34] P. Marshall., et. al, "EMCORE MTX8501/MRX8501 (12 x 1.25 Gbps Parallel Array Transmitter and Receiver Modules).," http://radhome.gsfc.nasa.gov/radhome/ papers/D020102 MTX8501 MRX8501.pdf; Feb. 2002

[35] S. Buchner, et al., "Proton Testing of Optocouplers (Micropac)," http://radhome.gsfc.nasa.gov/radhome/ papers/D040302_MII66099.pdf; Apr. 2002

[36] S. Buchner, et al., "Proton Testing of Micropac Optocouplers," http://radhome.gsfc.nasa.gov/radhome/ papers/1061002_MIl66099.pdf; June 2002

[37] J. Howard, et al., "Proton Testing of the Vectron CO-566 Oscillator," http://radhome.gsfc.nasa.gov/radhome/ papers/1060602_CO566.pdf; June 2003

[38] J. Howard, et al., "Proton Testing of the Vectron CO-718S Oscillator," http://radhome.gsfc.nasa.gov/radhome/ papers/1060602_CO718S.pdf; June 2003

[39] J. Howard, et al., "Proton Single Event Latchup Testing of the AD7664 Analog Devices Analog to Digital Converter," http://radhome.gsfc.nasa.gov/radhome/ papers/1021503_AD7664.pdf; Feb. 2003

[40] J. Howard, et al., "Proton and Heavy Ion Single Event Effects Testing of the Motorola ColdFire (MCF5307) Processor," http://radhome.gsfc.nasa.gov/radhome/ papers/1060602_TAMU081202_Coldfire_MCF5307.pdf; June 2003

[41] J. Howard, et al., "Proton Testing of the Hitachi SH-4 Processor," http://radhome.gsfc.nasa.gov/radhome/ papers/I060602_SH4.pdf; June 2002

[42] J. Howard, et al., "Proton Testing of the Zarlink Semiconductor GP2021 GPS 12-Channel Correlator," http://radhome.gsfc.nasa.gov/radhome/ papers/10602_GP2021.pdf; June 2002 